\title{
Effect of elliptic angle $\varphi$ on the existence and stability of libration points in restricted three-body problem in earth-moon system considering earth as an ellipsoid
}

\author{
M. Javed Idrisi*, Muhammad Amjad \\ Department of Mathematics Al-Falah University Faridabad (Haryana)-121004, India \\ *Corresponding authorE-mail:mjavedidrisi@gmail.com
}

\begin{abstract}
This paper deals with the existence and the stability of the earth-moon libration points in the restricted three-body problem. In this paper we have considered the bigger primary as an ellipsoid while the smaller one as a point-mass. This is observed that the collinear and non-collinear libration points exist only in the interval $0^{\circ}<\varphi<45^{\circ}$. There exist three collinear libration points and the noncollinear libration points are forming a right triangle with the primaries. Further observed that the libration points either collinear or non-collinear all are unstable in $0^{\circ}<\varphi<45^{\circ}$.
\end{abstract}

Keywords:Restricted Three-Body Problem; Libration Points; Linear Stability; Elliptic Integrals; Newton-Raphson Method.

\section{Introduction}

Restricted three-body problem is a well-known problem possesses five libration points; three are collinear and two are non-collinear. The non-collinear libration points form an equilateral triangle with the primaries and stable for a critical value of mass parameter $\mu_{\mathrm{c}}$ such that $\mu<\mu_{c}=0.0385208965 \ldots$ while the collinear libration points are unstable for all values of $\mu$. It has been studied by many scientists. In recent time, there are lots of papers in restricted three-body problem. Khanna and Bhatnagar (1999) have discussed the stationary solutions of the planar restricted three-body problem when the smaller primary is a triaxial rigid body with one of the axes as the axis of symmetry and its equatorial plane coinciding with the plane of motion. The bigger primary is taken as an oblate spheroid and its equatorial plane is also coinciding with the plane of motion. They have shown that there exist five libration points, two triangular and three collinear. The collinear points are unstable, while the triangular points are stable for the mass parameter 0 $\leq \mu<\mu_{\text {crit }}$ (the critical mass parameter) and the triangular points have long or short periodic elliptical orbits in the same range of $\mu$. Raheem and Singh (2006) have investigated the stability of equilibrium points under the influence of small perturbations in the coriolis and centrifugal forces, together with the effects of oblateness and radiation pressures of the primaries. They have found that the collinear points remain unstable while the triangular points are stable for $0 \leq \mu<\mu_{\mathrm{c}}$ and unstable for $\mu_{\mathrm{c}} \leq \mu \leq 1 / 2$, where $\mu_{\mathrm{c}}$ is the critical mass parameter depends upon the coriolis force, centrifugal force, oblateness and radiation pressure of the primaries.

Narayan and Kumar (2011) have studied the effects of the oblateness and the photogravitational of the bigger primary and the oblateness of the smaller primary on the location of the triangular Lagrangian equilibrium points in the elliptical restricted threebody problem. They observed that the range of stability decreases as the oblateness and the radiation pressure parameter increases. Idrisi and Taqvi (2013) have studied the restricted three-body problem in terms of elliptic integrals in which smaller primary is an ellipsoid and bigger one a point mass. They have determined the equations of motion of the infinitesimal mass and then investigated the collinear and non collinear libration points and their stability in the linear sense. They have also proven in their work that there exist five collinear libration points $\mathrm{L}_{\mathrm{i}}(\mathrm{i}=1,2, \ldots .5)$ and the non-collinear libration points lie on the arc of the unit circle centered at the bigger primary and both the collinear and noncollinear libration points are unstable for $0<\mu \leq 1 / 2$ and $0 \leq \varphi \leq$ $\pi / 2$. Singh et. al. (2013) have investigated the motion of an infinitesimal body in the generalized restricted three-body problem. It is generalized in the sense that both primaries are radiating, oblate bodies, together with the effect of gravitational potential from a belt. They have been found that, in addition to the usual five equilibrium points, there appear two new collinear points $\mathrm{L}_{\mathrm{n} 1}, \mathrm{~L}_{\mathrm{n} 2}$ due to the potential from the belt, and in the presence of all these perturbations, the equilibrium points $\mathrm{L}_{1}, \mathrm{~L}_{3}$ come nearer to the primaries; while $\mathrm{L}_{2}, \mathrm{~L}_{4}, \mathrm{~L}_{5}, \mathrm{~L}_{\mathrm{n} 1}$ move towards the less massive primary and $\mathrm{L}_{\mathrm{n} 2}$ moves away from it. The collinear equilibrium points remain unstable, while the triangular points are stable for $0<\mu$ $<\mu_{\mathrm{c}}$ and unstable for $\mu_{\mathrm{c}} \leq \mu \leq 1 / 2$. Abouelmagdet. al. (2013) have studied the generalized restricted three-body problem, generalized in the sense that the effects of oblateness of the three participating bodies as well as the small perturbations in the coriolis and centrifugal forces are considered. They have shown the existence of equilibrium points, their linear stability and the periodic orbits around these points under these effects. They have found that the positions of the collinear points and y-coordinate of the triangular points are not affected by the small perturbations in the coriolis force while $\mathrm{x}$-coordinate of the triangular points is neither affected by the small perturbations in the coriolis force nor the oblateness of the third body. Furthermore, the critical mass value and the elements of periodic orbits around the equilibrium points such as 
the semi-major and the semi-minor axes, the angular frequencies and corresponding periods may change by all the parameters of oblateness as well as the small perturbations in the coriolis and centrifugal forces. Reena Kumari et. al. (2014) have extended the basic model of the restricted four-body problem introducing two bigger dominant primaries $\mathrm{m}_{1}$ and $\mathrm{m}_{2}$ as oblate spheroids when masses of the two primary bodies $\left(\mathrm{m}_{2}\right.$ and $\left.\mathrm{m}_{3}\right)$ are equal. The aim of this study is to investigate the use of zero velocity surfaces and the Poincaré surfaces of section to determine the possible allowed boundary regions and the stability orbit of the equilibrium points. According to different values of Jacobi constant $\mathrm{C}$, we can determine a boundary region where the particle can move in possible permitted zones. The stability regions of the equilibrium points expanded due to presence of oblateness coefficient and various values of $C$, whereas for a certain range of $t(100 \leq t \leq 200)$, orbits form a shape of cote's spiral. For different values of oblateness parameters $A_{1}\left(0<A_{1}<1\right)$ and $A_{2}\left(0<A_{2}<1\right)$, they obtained two collinear and six non-collinear equilibrium points. The noncollinear equilibrium points are stable when the mass parameter $\mu$ lies in the interval $(0.0190637,0.647603)$. However, basins of attraction are constructed with the help of Newton Raphson method to demonstrate the convergence as well as divergence region of the equilibrium points. The nature of basins of attraction of the equilibrium points are less affected in presence and absence of oblateness coefficients $A_{1}$ and $A_{2}$ respectively in the proposed model. Idrisi and Taqvi (2014) have investigated the location and stability of the non-collinear libration points when both the primaries are ellipsoids and found that the non-collinear libration points exist only in the interval $52^{\circ}<\varphi<90^{\circ}$ and form an isosceles triangle with the primaries. Further they observed that the non collinear libration points are unstable in $52^{\circ}<\varphi<90^{\circ}$. Idrisi (2014) has found the location of the collinear and non-collinear libration points and their linear stability when smaller primary is an oblate spheroid. He has found that there exist three collinear libration points and the non-collinear libration points are lying on the circumference of the unit circle whose center is the bigger primary. The collinear and non-collinear libration points all are unstable in his case.

In this paper we have considered the smaller primary as a point mass and the bigger one an ellipsoid. Our aim is to show the existence of collinear and non-collinear libration points and then check their stability in linear sense for Earth-Moon system. There are 5 Sections in this paper. In Section 2, the equations of motion of the infinitesimal mass $\mathrm{m}_{3}$ have been determined. In Section 3, location of the collinear and non-collinear libration points have been investigated. In section 4 , we have checked the stability of the collinear and non-collinear libration points. In last section, we have discussed all the results.

\section{Equations of motion}

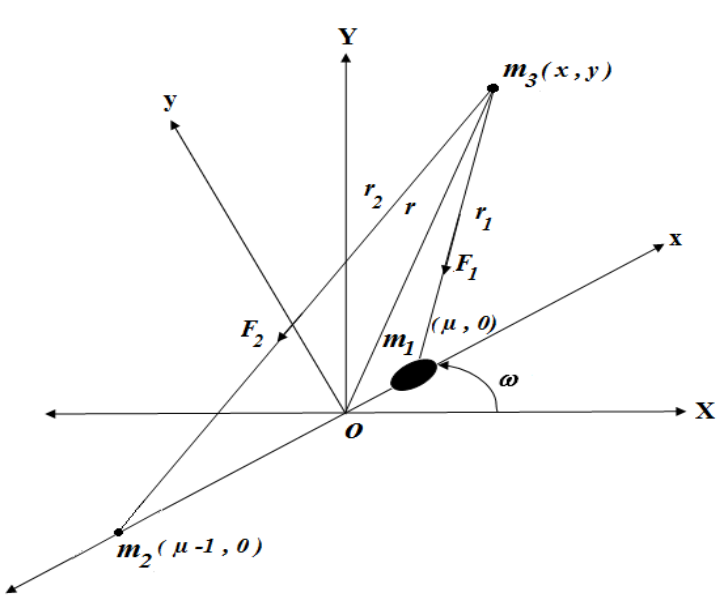

Fig. 1: The Configuration of the R3bp When $\mathrm{M}_{1}$ Is an Ellipsoid.

Let $\mathrm{m}_{1}$ be an ellipsoid whose axes are $\mathrm{a}, \mathrm{b}$ and $\mathrm{c}(\mathrm{a}>\mathrm{b}>\mathrm{c})$ and $\mathrm{m}_{2}$ a point mass $\left(\mathrm{m}_{1}>\mathrm{m}_{2}\right)$, are moving in the circular orbits around their center of mass $\mathrm{O}$. An infinitesimal mass $\mathrm{m}_{3}$ is moving in the plane of motion of $m_{1}$ and $m_{2}$. The distances of $m_{3}$ from $m_{1}, m_{2}$ and $\mathrm{O}$ are $\mathbf{r}_{1}, \mathbf{r}_{2}$ and $\mathbf{r}$ respectively. The principal axes of ellipsoid remains parallel to the synodic axes Oxyz throughout the motion and the equatorial plane of $m_{1}$ is coincided with the plane of motion of $\mathrm{m}_{1}$ and $\mathrm{m}_{2}$. Let the line joining $\mathrm{m}_{1}$ and $\mathrm{m}_{2}$ be taken as $\mathrm{X}$ axis and $\mathrm{O}$ their center of mass as origin. Let the line passing through $\mathrm{O}$ and perpendicular to $\mathrm{OX}$ and lying in the plane of motion $\mathrm{m}_{1}$ and $\mathrm{m}_{2}$ be the $\mathrm{Y}$-axis. Let us consider a synodic system of co-ordinates Oxyz initially coincide with the inertial system OX$\mathrm{YZ}$, rotating with angular velocity $\omega$ about $\mathrm{Z}$-axis (the $\mathrm{Z}$-axis is coincide with $\mathrm{Z}$-axis). We wish to find the equations of motion of $\mathrm{m}_{3}$ using the terminology of Szebehely (1967) in the synodic coordinate system and dimensionless variables i.e. the distance between the primaries is unity; the unit of time $t$ is such that the gravitational constant $\mathrm{G}=1$ and the sum of the masses of the primaries is unity i.e. $\mathrm{m}_{1}+\mathrm{m}_{2}=1$.

The potential of a homogeneous Ellipsoid at an external point $\mathrm{P}(\mathrm{x}$,

y, z) is given by (Byrd and Friedman, 1954)

$$
\begin{aligned}
& V=\frac{2 \pi \rho \alpha \beta \gamma}{\sqrt{\alpha^{2}-\gamma^{2}}}\left[\begin{array}{l}
\left(1-\frac{x^{2}}{\alpha^{2}-\beta^{2}}+\frac{y^{2}}{\alpha^{2}-\beta^{2}}\right) F(\varphi, k) \\
+\left(\frac{x^{2}}{\alpha^{2}-\beta^{2}}+\frac{\left(\gamma^{2}-\alpha^{2}\right) y^{2}}{\left(\alpha^{2}-\beta^{2}\right)\left(\beta^{2}-\gamma^{2}\right)}+\frac{z^{2}}{\beta^{2}-\gamma^{2}}\right) E(\varphi, k)
\end{array}\right. \\
& \left.+\left(\frac{\gamma^{2}+\gamma_{1}}{\beta^{2}-\gamma^{2}} y^{2}-\frac{\beta^{2}+\gamma_{1}}{\beta^{2}-\gamma^{2}} z^{2}\right) \sqrt{\frac{\alpha^{2}-\gamma^{2}}{\left(\alpha^{2}+\gamma_{1}\right)\left(\beta^{2}+\gamma_{1}\right)\left(\gamma^{2}+\gamma_{1}\right)}}\right]
\end{aligned}
$$

$\alpha, \beta, \gamma=$ semi-axes of the ellipsoid,

$\rho=$ the density of the ellipsoid,

$\gamma_{1}=$ largest root of the confocal ellipsoid

$\frac{x^{2}}{\alpha^{2}+\gamma_{1}}+\frac{y^{2}}{\beta^{2}+\gamma_{1}}+\frac{z^{2}}{\gamma^{2}+\gamma_{1}}=1$,

Where

$F(\varphi, k)=$ Elliptic Integral of First Kind $=\int_{0}^{\varphi} \frac{d \theta}{\sqrt{1-k^{2} \sin ^{2} \theta}}$

$E(\varphi, k)=$ Elliptic Integral of Second Kind $=\int_{0}^{\varphi} \sqrt{1-k^{2} \sin ^{2} \theta} d \theta$,

$\varphi=\sin ^{-1} \sqrt{\frac{\alpha^{2}-\gamma^{2}}{\gamma_{1}+\alpha^{2}}}, 0 \leq \varphi \leq \frac{\pi}{2}$

and

$k=\sqrt{\frac{\alpha^{2}-\beta^{2}}{\alpha^{2}-\gamma^{2}}}, 0 \leq k^{2} \leq 1$ i.e. $-1 \leq k \leq 1$.

The equations of motion of the infinitesimal mass $m_{3}$ in terms of elliptic integrals of first and second kind are given by: 


$$
\begin{aligned}
& \ddot{x}-2 n \dot{y}=n^{2} x-3(1-\mu)(x-\mu)\left[\frac{E(\varphi, k)-F(\varphi, k)}{p_{6} p_{8}}\right. \\
& -\left\{1-k^{2} \sin ^{2} \varphi \frac{(x-\mu)^{2}}{p_{6}}+\left(\frac{1}{p_{6}}+\frac{1-k^{2} \sin ^{2} \varphi}{p_{9}}\right) y^{2}\right\} \\
& \times \frac{\left(\lambda+p_{3}\right)}{2\left(\lambda+a^{2}\right)\left(2 \lambda+p_{1}-r_{1}^{2}\right) \sqrt{\lambda^{2}} \sqrt{1-k^{2} \sin ^{2} \varphi}} \\
& \left.-\frac{\left(\lambda^{2}+2 c^{2} \lambda+p_{11}\right)\left(\lambda+p_{3}\right) y^{2}}{2 p_{7}\left(2 \lambda+p_{1}-r_{1}^{2}\right) \sqrt{\lambda+c^{2}}\left(\lambda^{2}+p_{5} \lambda+p_{10}\right)^{\frac{3}{2}}}\right] \\
& -\frac{\mu}{r_{2}^{3}}(x+1-\mu)
\end{aligned}
$$

and

$$
\begin{aligned}
& p_{10}=a^{2} b^{2}, \\
& p_{11}=p_{10}-c^{2} p_{5}, \\
& \varphi=\sin ^{-1} \sqrt{\frac{a^{2}-c^{2}}{\lambda+a^{2}}}, 0 \leq \varphi \leq \frac{\pi}{2}, \\
& k=\sqrt{\frac{a^{2}-b^{2}}{a^{2}-c^{2}}}, 0 \leq k^{2} \leq 1 \text { i.e. }-1 \leq k \leq 1,
\end{aligned}
$$

$$
\begin{aligned}
& \ddot{y}+2 n \dot{x}=n^{2} y-3(1-\mu) y\left[\frac { 1 } { p _ { 8 } } \left(\frac{E(\varphi, k)}{\left.p_{9}+\frac{F(\varphi, k)}{p_{6}}\right)}\right.\right. \\
& -\left\{1-k^{2} \sin ^{2} \varphi \frac{(x-\mu)^{2}}{p_{6}}+\left(\frac{1}{p_{6}}+\frac{1-k^{2} \sin ^{2} \varphi}{p_{9}}\right) y^{2}\right\} \\
& \times \frac{\left(\lambda+p_{4}\right)}{2\left(\lambda+a^{2}\right)\left(2 \lambda+p_{1}-r_{1}^{2}\right) \sqrt{\lambda+c^{2}} \sqrt{1-k^{2} \sin ^{2} \varphi}} \\
& -\frac{\left(\lambda^{2}+2 c^{2} \lambda+p_{11}\right)\left(\lambda+p_{4}\right) y^{2}}{2 p_{7}\left(2 \lambda+p_{1}-r_{1}^{2}\right) \sqrt{\lambda+c^{2}}\left(\lambda^{2}+p_{5} \lambda+p_{10}\right)^{\frac{3}{2}}} \\
& \left.+\frac{\sqrt{\lambda+c^{2}}}{p_{7} \sqrt{\lambda^{2}+p_{5} \lambda+p_{10}}}\right]-\frac{\mu}{r_{2}^{3}} y
\end{aligned}
$$

$$
\begin{aligned}
& r_{1}=\sqrt{(x-\mu)^{2}+y^{2}}, \\
& r_{2}=\sqrt{(x+1-\mu)^{2}+y^{2}} .
\end{aligned}
$$

The mean-motion of the primaries is given by

$$
n=\left[3\left\{\frac{\left(\begin{array}{l}
\left.1-\frac{k^{2} \sin ^{2} \varphi^{\prime}}{p_{6}}\right) \\
\frac{2\left(\lambda^{\prime}+a^{2}\right)\left(2 \lambda^{\prime}+p_{1}-1\right) \sqrt{\lambda^{\prime}+c^{2}} \sqrt{1-k^{2} \sin ^{2} \varphi^{\prime}}}{-\frac{E\left(\varphi^{\prime}, k\right)-F\left(\varphi^{\prime}, k\right)}{p_{6} p_{8}}}
\end{array}\right]}{]^{\frac{1}{2}}}\right]^{\frac{1}{2}}\right.
$$

where

$$
\begin{aligned}
& \lambda^{\prime}=\frac{1-p_{1}+\sqrt{\left(1-p_{1}\right)^{2}+4\left(p_{3}-p_{2}\right)}}{2}, \\
& \varphi^{\prime}=\sin ^{-1} \sqrt{\frac{a^{2}-c^{2}}{\lambda^{\prime}+a^{2}}}, 0 \leq \varphi^{\prime} \leq \frac{\pi}{2} .
\end{aligned}
$$

From the astrophysical data:

Mass of Moon $\left(\mathrm{m}_{2}\right)=7.3477 \times 10^{22} \mathrm{~kg}$,

Mass of Earth $\left(\mathrm{m}_{1}\right)=5.9742 \times 10^{24} \mathrm{~kg}$,

Axes of the Earth: $a=6378.140 \mathrm{~km}, \mathrm{~b}=6368 \mathrm{~km}$ and $\mathrm{c}=$ $6356.755 \mathrm{~km}$

Mean distance of Moon from the Earth $=384,400 \mathrm{~km}$.

In dimensionless system:

$\mathrm{m}_{1}+\mathrm{m}_{2}=1$ unit i.e. $1 \mathrm{~kg}=1.65353 \times 10^{-25}$ unit.

Therefore,

$\mu=\frac{m_{2}}{m_{1}+m_{2}}=0.0121496$ 
Distance between the primaries $=1$ unit i.e. $1 \mathrm{~km}=2.60146 \times 10^{-6}$ unit,

Thus, $\mathrm{a}=0.0165925, \mathrm{~b}=0.0165661$ and $\mathrm{c}=0.0165368$.

From Eqn. (3), mean-motion of the primaries is $n=1.20494$.

$k=\sqrt{\frac{a^{2}-b^{2}}{a^{2}-c^{2}}}=0.688757$,

$\varphi=\sin ^{-1} \sqrt{\frac{a^{2}-c^{2}}{\lambda+a^{2}}} \Rightarrow \lambda=a^{2}\left(\operatorname{cosec}^{2} \varphi-1\right)-a^{2}, 0 \leq \varphi \leq \frac{\pi}{2}$.

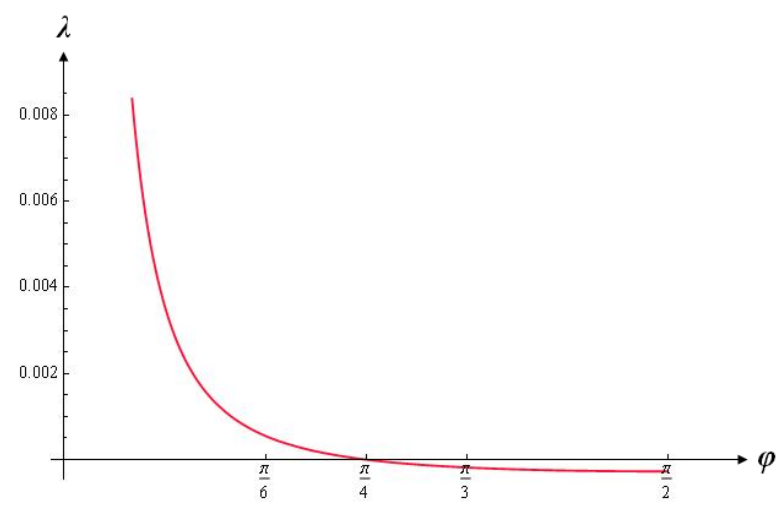

Fig. 2: $\varphi$ versus $\lambda$ in Earth-Moon System.

From the Fig. 2, this is observed that as $\varphi \rightarrow \pi / 4, \lambda \rightarrow 0$, as $\varphi \rightarrow 0$, $\lambda \rightarrow \infty$ and in the interval $\pi / 4 \leq \varphi \leq \pi / 2, \lambda$ is negative. Thus, in Earth-Moon system $\varphi$ is valid only in the interval $0<\varphi<\pi / 4$.

\section{Libration points}

At the libration points $\dot{x}=0, \dot{y}=0, \ddot{x}=0, \ddot{y}=0$ therefore Eqns. (1) and (2) become

$$
\begin{aligned}
& n^{2} x-3(1-\mu)(x-\mu)\left[\frac{E(\varphi, k)-F(\varphi, k)}{p_{6} p_{8}}\right. \\
& -\left\{1-k^{2} \sin ^{2} \varphi \frac{(x-\mu)^{2}}{p_{6}}+\left(\frac{1}{p_{6}}+\frac{1-k^{2} \sin ^{2} \varphi}{p_{9}}\right) y^{2}\right\} \\
& \times \frac{\left(\lambda+p_{3}\right)}{2\left(\lambda+a^{2}\right)\left(2 \lambda+p_{1}-r_{1}^{2}\right) \sqrt{\lambda_{+c^{2}}} \sqrt{1-k^{2} \sin ^{2} \varphi}} \\
& \left.-\frac{\left(\lambda^{2}+2 c^{2} \lambda+p_{11}\right)\left(\lambda+p_{3}\right) y^{2}}{2 p_{7}\left(2 \lambda+p_{1}-r_{1}^{2}\right) \sqrt{\lambda+c^{2}}\left(\lambda^{2}+p_{5} \lambda+p_{10}\right)^{\frac{3}{2}}}\right] \\
& -\frac{\mu}{r_{2}^{3}}(x+1-\mu)=0
\end{aligned}
$$

and

$$
\begin{aligned}
& n^{2} y-3(1-\mu) y\left[\frac{1}{p_{8}}\left(\frac{E(\varphi, k)}{p_{9}}+\frac{F(\varphi, k)}{p_{6}}\right)\right. \\
& -\left\{1-k^{2} \sin ^{2} \varphi \frac{(x-\mu)^{2}}{p_{6}}+\left(\frac{1}{p_{6}}+\frac{1-k^{2} \sin ^{2} \varphi}{p_{9}}\right) y^{2}\right\} \\
& \times \frac{\left(\lambda+p_{4}\right)}{2\left(\lambda+a^{2}\right)\left(2 \lambda+p_{1}-r_{1}^{2}\right) \sqrt{\lambda+c^{2}} \sqrt{1-k^{2} \sin ^{2} \varphi}} \\
& -\frac{\left(\lambda^{2}+2 c^{2} \lambda+p_{11}\right)\left(\lambda+p_{4}\right) y^{2}}{2 p_{7}\left(2 \lambda+p_{1}-r_{1}^{2}\right) \sqrt{\lambda+c^{2}}\left(\lambda^{2}+p_{5} \lambda+p_{10}\right)^{\frac{3}{2}}} \\
& \left.+\frac{\sqrt{\lambda^{2} c^{2}}}{p_{7} \sqrt{\lambda^{2}+p_{5} \lambda+p_{10}}}\right]-\frac{\mu}{r_{2}^{3}} y=0
\end{aligned}
$$

\subsection{Collinear libration points}

The collinear libration points are the solution of the Equations (5) and (6) when $\mathrm{y}=0$ i.e.

$$
\begin{aligned}
& n^{2} x-3(1-\mu)(x-\mu)\left[\frac{E(\varphi, k)-F(\varphi, k)}{p_{6} p_{8}}\right. \\
& -\left\{1-k^{2} \sin ^{2} \varphi \frac{(x-\mu)^{2}}{p_{6}}\right\} \\
& \left.\times \frac{\left(\lambda+p_{3}\right)}{2\left(\lambda+a^{2}\right)\left(2 \lambda+p_{1}-(x-\mu)^{2}\right) \sqrt{\lambda+c^{2}} \sqrt{1-k^{2} \sin ^{2} \varphi}}\right] \\
& -\frac{\mu}{(x+1-\mu)^{2}}=0
\end{aligned}
$$

The Eqn. (6) is a fifth degree equation in $\mathrm{x}$, depending upon $\varphi$. On solving Eqn. (6) for $\mathrm{a}=0.0165925, \mathrm{~b}=0.0165661, \mathrm{c}=0.0165368$ and $\mu=0.0121496$ in $0<\varphi<\pi / 4$ we get three values of $x$, these values are given in Table 1 .

As shown in the Fig. 3, there exist three collinear libration points $\mathrm{L}_{\mathrm{i}}(\mathrm{i}=1,2,3)$. The first libration point $\mathrm{L}_{1}$ lies between $\mathrm{m}_{2}$ and the center of mass $\mathrm{O}$, as $\varphi \rightarrow 9^{\circ}, \mathrm{L}_{1} \rightarrow \mathrm{O}$ and in $9^{\circ} \leq \varphi<45^{\circ}$ it moves away from the center of mass. The center of mass of bigger primary $\mathrm{m}_{1}$ i.e. ellipsoid behaves as a second libration point $\mathrm{L}_{2}$ and remains stationary in $0^{\circ}<\varphi<45^{\circ}$, the third libration point $\mathrm{L}_{3}$ lies at the right of $\mathrm{m}_{1}$ and move towards the center of mass in $0^{\circ}<\varphi<45^{\circ}$. 
Table 1: Collinear Libration Points inEarth-Moon System

\begin{tabular}{|c|c|c|c|c|c|c|c|}
\hline$\varphi^{\circ}$ & $\mathrm{L}_{1}(\mathrm{x})$ & $\mathrm{L}_{2}(\mathrm{x})$ & $\mathrm{L}_{3}(\mathrm{x})$ & $\varphi^{\circ}$ & $\mathrm{L}_{1}(\mathrm{x})$ & $\mathrm{L}_{2}(\mathrm{x})$ & $\mathrm{L}_{3}(\mathrm{x})$ \\
\hline 1 & -0.0832616 & 0.0121484 & 0.1075610 & 23 & 0.0073625 & 0.0121496 & 0.0169367 \\
\hline 2 & -0.0360226 & 0.0121495 & 0.0603218 & 24 & 0.0075515 & 0.0121496 & 0.0167477 \\
\hline 3 & -0.0204022 & 0.0121496 & 0.0447014 & 25 & 0.0077255 & 0.0121496 & 0.0165737 \\
\hline 4 & -0.0126271 & 0.0121496 & 0.0369263 & 26 & 0.0078860 & 0.0121496 & 0.0164132 \\
\hline 5 & -0.0079555 & 0.0121496 & 0.0322547 & 27 & 0.0080346 & 0.0121496 & 0.0162646 \\
\hline 6 & -0.0048199 & 0.0121496 & 0.0291191 & 28 & 0.0081725 & 0.0121496 & 0.0161267 \\
\hline 7 & -0.0025573 & 0.0121496 & 0.0268565 & 29 & 0.0083008 & 0.0121496 & 0.0159984 \\
\hline 8 & -0.0008403 & 0.0121496 & 0.0251394 & 30 & 0.0084205 & 0.0121496 & 0.0158787 \\
\hline 9 & 0.00051114 & 0.0121496 & 0.0237881 & 31 & 0.0085323 & 0.0121496 & 0.0157669 \\
\hline 10 & 0.00160438 & 0.0121496 & 0.0226948 & 32 & 0.0086369 & 0.0121496 & 0.0156622 \\
\hline 11 & 0.00250789 & 0.0121496 & 0.0217913 & 33 & 0.0087352 & 0.0121496 & 0.0155641 \\
\hline 12 & 0.00326751 & 0.0121496 & 0.0210317 & 34 & 0.0088275 & 0.0121496 & 0.0154717 \\
\hline 13 & 0.00391519 & 0.0121496 & 0.0203840 & 35 & 0.0089143 & 0.0121496 & 0.0153849 \\
\hline 14 & 0.00447397 & 0.0121496 & 0.0198252 & 36 & 0.0089962 & 0.0121496 & 0.0153031 \\
\hline 15 & 0.00496093 & 0.0121496 & 0.0193383 & 37 & 0.0090735 & 0.0121496 & 0.0152257 \\
\hline 16 & 0.0053889 & 0.0121496 & 0.018911 & 38 & 0.0091466 & 0.0121496 & 0.0151526 \\
\hline 17 & 0.0057681 & 0.0121496 & 0.018531 & 39 & 0.0092158 & 0.0121496 & 0.0150834 \\
\hline 18 & 0.0061062 & 0.0121496 & 0.018193 & 40 & 0.0092814 & 0.0121496 & 0.0150178 \\
\hline 19 & 0.0064095 & 0.0121496 & 0.017889 & 41 & 0.0093437 & 0.0121496 & 0.0149555 \\
\hline 20 & 0.0066829 & 0.0121496 & 0.017616 & 42 & 0.0094028 & 0.0121496 & 0.0148964 \\
\hline 21 & 0.0069308 & 0.0121496 & 0.017368 & 43 & 0.0094591 & 0.0121496 & 0.0148401 \\
\hline 22 & 0.0071564 & 0.0121496 & 0.017143 & 44 & 0.0095127 & 0.0121496 & 0.0147865 \\
\hline
\end{tabular}

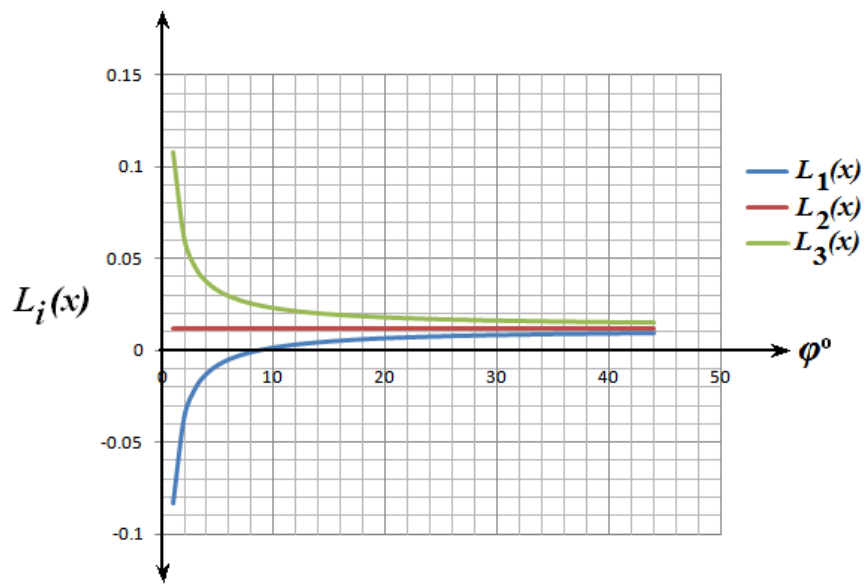

Fig. 3: $\Phi$ versus $L_{i}(X), I=1,2,3$.

\subsection{Non-collinear libration points}

The non-collinear libration points are the solution of the Eqns. (1) and (2) when $\mathrm{y} \neq 0$ i.e.

$$
\begin{aligned}
& n^{2} x-3(1-\mu)(x-\mu)\left[\frac{E(\varphi, k)-F(\varphi, k)}{p_{6} p_{8}}\right. \\
& -\left\{1-k^{2} \sin ^{2} \varphi \frac{(x-\mu)^{2}}{p_{6}}+\left(\frac{1}{p_{6}}+\frac{1-k^{2} \sin ^{2} \varphi}{p_{9}}\right) y^{2}\right\} \\
& \times \frac{\left(\lambda+p_{3}\right)}{2\left(\lambda+a^{2}\right)\left(2 \lambda+p_{1}-r_{1}^{2}\right) \sqrt{\lambda_{+c^{2}}} \sqrt{1-k^{2} \sin ^{2} \varphi}} \\
& \left.-\frac{\left(\lambda^{2}+2 c^{2} \lambda+p_{11}\right)\left(\lambda+p_{3}\right) y^{2}}{2 p_{7}\left(2 \lambda+p_{1}-r_{1}^{2}\right) \sqrt{\lambda+c^{2}}\left(\lambda^{2}+p_{5} \lambda+p_{10}\right)^{\frac{3}{2}}}\right] \\
& -\frac{\mu}{3}(x+1-\mu)=0 \\
& r_{2}
\end{aligned}
$$

$$
\begin{aligned}
& n^{2}-3(1-\mu)\left[\frac{1}{p_{8}}\left(\frac{E(\varphi, k)}{p_{9}}+\frac{F(\varphi, k)}{p_{6}}\right)\right. \\
& -\left\{1-k^{2} \sin ^{2} \varphi \frac{(x-\mu)^{2}}{p_{6}}+\left(\frac{1}{p_{6}}+\frac{1-k^{2} \sin ^{2} \varphi}{p_{9}}\right) y^{2}\right\} \\
& \times \frac{\left(\lambda+p_{4}\right)}{2\left(\lambda+a^{2}\right)\left(2 \lambda+p_{1}-r_{1}^{2}\right) \sqrt{\lambda+c^{2}} \sqrt{1-k^{2} \sin ^{2} \varphi}} \\
& -\frac{\left(\lambda^{2}+2 c^{2} \lambda+p_{11}\right)\left(\lambda+p_{4}\right) y^{2}}{2 p_{7}\left(2 \lambda+p_{1}-r_{1}^{2}\right) \sqrt{\lambda+c^{2}}\left(\lambda^{2}+p_{5} \lambda+p_{10}\right)^{\frac{3}{2}}} \\
& \left.+\frac{\sqrt{\lambda+c^{2}}}{p_{7} \sqrt{\lambda^{2}+p_{5} \lambda+p_{10}}}\right]-\frac{\mu}{r_{2}^{3}}=0
\end{aligned}
$$

On solving the Eqns. (7) and (8) simultaneously for $0^{\circ}<\varphi<45^{\circ}$ by Newton-Raphson Method, taking the initial conditions: $\mathrm{x}_{0}=$ $\mu-1 / 2$ and $y_{0}= \pm \sqrt{3} / 2$, we get the co-ordinates of non-collinear libration points $\mathrm{L}_{4,5}$ in Earth-Moon system given in Table 2 . 
Table 2:Non-Collinear Libration Points in Earth-Moon System

\begin{tabular}{llllll}
\hline$\varphi^{\circ}$ & $\mathrm{x}$ & $\pm \mathrm{y}$ & $\varphi^{\mathrm{o}}$ & $\mathrm{x}$ & $\pm \mathrm{y}$ \\
\hline 1 & 0.0121472 & $\mp_{0.0181461}$ & 23 & 0.0121496 & $\mp_{0.0000330987}$ \\
2 & 0.0121493 & $\mp_{0.00457585}$ & 24 & 0.0121496 & $\mp_{0.0000304438}$ \\
3 & 0.0121495 & $\mp 0.00201824$ & 25 & 0.0121496 & $\mp 0.0000281027$ \\
4 & 0.0121496 & $\mp 0.00112455$ & 26 & 0.0121496 & $\mp 0.0000260278$ \\
5 & 0.0121496 & $\mp 0.000713569$ & 27 & 0.0121496 & $\mp 0.0000241803$ \\
6 & 0.0121496 & $\mp 0.000492075$ & 28 & 0.0121496 & $\mp 0.0000225283$ \\
7 & 0.0121496 & $\mp 0.000359556$ & 29 & 0.0121496 & $\mp 0.0000210451$ \\
8 & 0.0121496 & $\mp 0.000274146$ & 30 & 0.0121496 & $\mp 0.0000197084$ \\
9 & 0.0121496 & $\mp 0.000215941$ & 31 & 0.0121496 & $\mp 0.0000184998$ \\
10 & 0.0121496 & $\mp 0.000174517$ & 32 & 0.0121496 & $\mp 0.0000174035$ \\
11 & 0.0121496 & $\mp 0.000143999$ & 33 & 0.0121496 & $\mp 0.0000164059$ \\
12 & 0.0121496 & $\mp 0.000120869$ & 34 & 0.0121496 & $\mp 0.0000154957$ \\
13 & 0.0121496 & $\mp 0.000102922$ & 35 & 0.0121496 & $\mp 0.0000146631$ \\
14 & 0.0121496 & $\mp 0.0000887176$ & 36 & 0.0121496 & $\mp 0.0000138994$ \\
15 & 0.0121496 & $\mp 0.0000772824$ & 37 & 0.0121496 & $\mp 0.0000131974$ \\
16 & 0.0121496 & $\mp 0.0000679405$ & 38 & 0.0121496 & $\mp 0.0000125506$ \\
17 & 0.0121496 & $\mp 0.0000602102$ & 39 & 0.0121496 & $\mp 0.0000119535$ \\
18 & 0.0121496 & $\mp 0.0000537409$ & 40 & 0.0121496 & $\mp 0.0000114013$ \\
19 & 0.0121496 & $\mp 0.0000482725$ & 41 & 0.0121496 & $\mp 0.0000108895$ \\
20 & 0.0121496 & $\mp 0.0000436085$ & 42 & 0.0121496 & $\mp 0.0000104144$ \\
21 & 0.0121496 & $\mp 0.0000395986$ & 43 & 0.0121496 & $\mp 0.0000099726$ \\
22 & 0.0121496 & $\mp 0.0000361259$ & 44 & 0.0121496 & $\mp 0.0000095612$ \\
\hline & & & & &
\end{tabular}

From the Table 2, it is observed the non-collinear libration points are forming a right angled-triangle with the primaries, right angled at bigger primary (Earth) for $0^{\circ}<\varphi<45^{\circ}$ and as $\varphi$ increases the shape of right triangle reduces. Therefore, on the basis of the results obtained we can say that the non-collinear libration points in Earth-Moon system lie in the neighborhood of Earth (Fig. 4).

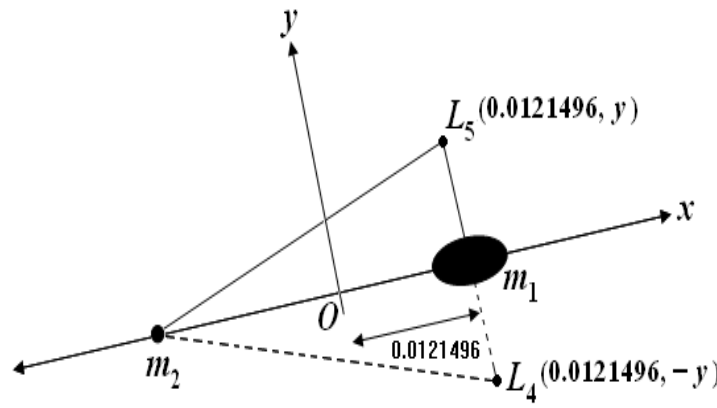

\section{Stability of the libration points}

The equations of the motion of the infinitesimal mass are

$$
\begin{aligned}
& \ddot{x}-2 n \dot{y}=\Omega_{x} \\
& \ddot{y}+2 n \dot{x}=\Omega_{y}
\end{aligned}
$$

To study the possible motion of the infinitesimal mass around the libration points let the coordinates of these points are $\left(\mathrm{x}_{0}, \mathrm{y}_{0}\right)$. If we give small displacement $(\alpha, \beta)$ to $\left(\mathrm{x}_{0}, \mathrm{y}_{0}\right)$, the variation $\alpha$ and $\beta$ can be written as: $\alpha=\mathrm{x}-\mathrm{x}_{0}$ and $\beta=\mathrm{y}-\mathrm{y}_{0}$ and the equations of the motion become

$$
\begin{gathered}
\ddot{\alpha}-2 n \dot{\beta}=\Omega_{x}\left(x_{0}+\alpha, y_{0}+\beta\right)=\alpha \Omega_{x x}+\beta \Omega x y, \\
\ddot{\beta}+2 n \dot{\alpha}=\Omega_{y}\left(x_{0}+\alpha, y_{0}+\beta\right)=\alpha \Omega_{y x}+\beta \Omega y y,
\end{gathered}
$$

where ' $\mathrm{o}$ ' indicates that the partial derivatives are to be calculated at the libration points under consideration.

The characteristic equation of the Equations (8) is given by

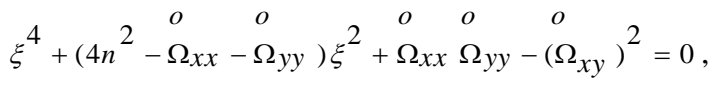

which is a fourth degree equation in $\xi$. The libration point $\left(\mathrm{x}_{0}, \mathrm{y}_{0}\right)$ is said to be stable if all the four roots of the Equation (11) are either negative real numbers or pure imaginary.

Note: The values of $\Omega_{\mathrm{xx}}, \Omega_{\mathrm{xy}}$ and $\Omega_{\mathrm{yy}}$ are given in Appendix I.

\subsection{Stability of collinear libration points}

At the collinear libration points $\mathrm{y}=0$. Therefore, $\mathrm{A}_{5}=\mathrm{A}_{6}=\mathrm{A}_{7}=$ o

$\mathrm{A}_{8}=0$ i.e. $\Omega_{x y}=0$. Thus the roots of the characteristic Eqn.

\begin{tabular}{|c|c|c|c|c|c|}
\hline$\varphi^{\circ}$ & $\mathrm{L}_{1}(\mathrm{x})$ & $\Omega_{\mathrm{xx}}^{\mathrm{o}}$ & $\Omega_{\mathrm{yy}}^{\mathrm{o}}$ & $\xi_{1,2}$ & $\xi_{3,4}$ \\
\hline 1 & -0.0832616 & 73971.1 & -2321.14 & \pm 271.966 & $\pm 48.18 \mathrm{i}$ \\
\hline 2 & -0.0360226 & 502793 & -18575.4 & \pm 709.075 & $\pm 136.292 \mathrm{i}$ \\
\hline 3 & -0.0204022 & $1.37909 \times 10^{6}$ & -62715.1 & \pm 1174.35 & $\pm 250.43 \mathrm{i}$ \\
\hline 4 & -0.0126271 & $2.66487 \times 10^{6}$ & -148697.1 & \pm 1632.44 & $\pm 385.612 \mathrm{i}$ \\
\hline 5 & -0.0079555 & $4.36679 \times 10^{6}$ & -290447.1 & \pm 2089.69 & $\pm 538.932 \mathrm{i}$ \\
\hline 6 & -0.0048199 & $6.55878 \times 10^{6}$ & -501827.1 & \pm 2561.01 & $\pm 708.398 \mathrm{i}$ \\
\hline 7 & -0.0025573 & $9.35025 \times 10^{6}$ & -796700.1 & \pm 3057.82 & $\pm 892.581 \mathrm{i}$ \\
\hline 8 & -0.0008403 & $1.28589 \times 10^{7}$ & $-1.18877 \times 10^{6}$ & \pm 3585.94 & $\pm 1090.31 \mathrm{i}$ \\
\hline 9 & 0.00051114 & $1.72034 \times 10^{7}$ & $-1.69163 \times 10^{6}$ & \pm 4147.71 & $\pm 1300.63 \mathrm{i}$ \\
\hline 10 & 0.00160438 & $2.2504 \times 10^{7}$ & $-2.31892 \times 10^{6}$ & \pm 4743.83 & $\pm 1522.8 \mathrm{i}$ \\
\hline 11 & 0.00250789 & $2.8878 \times 10^{7}$ & $-3.08407 \times 10^{6}$ & \pm 5373.83 & $\pm 1756.15 \mathrm{i}$ \\
\hline 12 & 0.00326751 & $3.64431 \times 10^{7}$ & $-4.00039 \times 10^{6}$ & \pm 6036.81 & $\pm 2000.1 \mathrm{i}$ \\
\hline 13 & 0.00391519 & $4.53182 \times 10^{7}$ & $-5.0811 \times 10^{6}$ & \pm 6731.88 & $\pm 2254.13 \mathrm{i}$ \\
\hline 14 & 0.00447397 & $5.56237 \times 10^{7}$ & $-6.33927 \times 10^{6}$ & \pm 7458.13 & $\pm 2517.79 \mathrm{i}$ \\
\hline 15 & 0.00496093 & $6.74806 \times 10^{7}$ & $-7.78774 \times 10^{6}$ & \pm 8214.66 & $\pm 2790.65 \mathrm{i}$ \\
\hline 16 & 0.0053889 & $8.10249 \times 10^{7}$ & $-9.44005 \times 10^{6}$ & \pm 9001.38 & $\pm 3072.47 \mathrm{i}$ \\
\hline 17 & 0.0057681 & $9.63575 \times 10^{7}$ & $-1.13069 \times 10^{7}$ & \pm 9816.18 & $\pm 3362.58 \mathrm{i}$ \\
\hline 18 & 0.0061062 & $1.13628 \times 10^{8}$ & $-1.3402 \times 10^{7}$ & \pm 10659.6 & $\pm 3660.88 \mathrm{i}$ \\
\hline 19 & 0.0064095 & $1.32962 \times 10^{8}$ & $-1.57371 \times 10^{7}$ & \pm 11530.9 & $\pm 3967.01 \mathrm{i}$ \\
\hline 20 & 0.0066829 & $1.54535 \times 10^{8}$ & $-1.83266 \times 10^{7}$ & \pm 12431.2 & $\pm 4280.96 \mathrm{i}$ \\
\hline 21 & 0.0069308 & $1.78416 \times 10^{8}$ & $-2.11771 \times 10^{7}$ & \pm 13357.2 & $\pm 4601.85 \mathrm{i}$ \\
\hline 22 & 0.0071564 & $2.04798 \times 10^{8}$ & $-2.43033 \times 10^{7}$ & \pm 14310.8 & $\pm 4929.84 \mathrm{i}$ \\
\hline 23 & 0.0073625 & $2.3385 \times 10^{8}$ & $-2.77178 \times 10^{7}$ & \pm 15292.2 & $\pm 5264.77 \mathrm{i}$ \\
\hline 24 & 0.0075515 & $2.65722 \times 10^{8}$ & $-3.14309 \times 10^{7}$ & \pm 16301.1 & $\pm 5606.32 \mathrm{i}$ \\
\hline 25 & 0.0077255 & $3.00518 \times 10^{8}$ & $-3.54488 \times 10^{7}$ & \pm 17335.4 & $\pm 5953.89 \mathrm{i}$ \\
\hline 26 & 0.0078860 & $3.38531 \times 10^{8}$ & $-3.97909 \times 10^{7}$ & \pm 18399.2 & $\pm 6308.01 \mathrm{i}$ \\
\hline 27 & 0.0080346 & $3.79853 \times 10^{8}$ & $-4.44607 \times 10^{7}$ & \pm 19489.8 & $\pm 6667.89 \mathrm{i}$ \\
\hline 28 & 0.0081725 & $4.24712 \times 10^{8}$ & $-4.94711 \times 10^{7}$ & \pm 20608.5 & $\pm 7033.57 \mathrm{i}$ \\
\hline
\end{tabular}
(11) i.e. $\xi_{i}(i=1,2,3,4)$ for $\mathrm{L}_{i}(i=1,2,3)$ are given in Table 3,4 and 5 respectively.

Fig. 4: Location of Non-Collinear Libration Points in Earth-Moon System.

Table 3: Stability of $\mathrm{L}$ 


\begin{tabular}{|c|c|c|c|c|c|}
\hline 29 & 0.0083008 & $4.73294 \times 10^{8}$ & $-5.48305 \times 10^{7}$ & \pm 21755.3 & $\pm 7404.76 \mathrm{i}$ \\
\hline 30 & 0.0084205 & $5.25732 \times 10^{8}$ & $-6.05427 \times 10^{7}$ & \pm 22928.8 & $\pm 7780.92 \mathrm{i}$ \\
\hline 31 & 0.0085323 & $5.82401 \times 10^{8}$ & $-6.66277 \times 10^{7}$ & \pm 24133.1 & $\pm 8162.58 \mathrm{i}$ \\
\hline 32 & 0.0086369 & $6.43612 \times 10^{8}$ & $-7.30996 \times 10^{7}$ & \pm 25369.5 & $\pm 8549.83 \mathrm{i}$ \\
\hline 33 & 0.0087352 & $7.09162 \times 10^{8}$ & $-7.99336 \times 10^{7}$ & \pm 26630.1 & $\pm 8940.56 \mathrm{i}$ \\
\hline 34 & 0.0088275 & $7.7977 \times 10^{8}$ & $-8.71705 \times 10^{7}$ & \pm 27924.4 & $\pm 9336.51 \mathrm{i}$ \\
\hline 35 & 0.0089143 & $8.55785 \times 10^{8}$ & $-9.48224 \times 10^{7}$ & \pm 29253.8 & $\pm 9737.68 \mathrm{i}$ \\
\hline 36 & 0.0089962 & $9.37166 \times 10^{8}$ & $-1.02871 \times 10^{8}$ & \pm 30613.2 & $\pm 10142.5 \mathrm{i}$ \\
\hline 37 & 0.0090735 & $1.02449 \times 10^{9}$ & $-1.11342 \times 10^{8}$ & \pm 32007.6 & $\pm 10551.9 \mathrm{i}$ \\
\hline 38 & 0.0091466 & $1.11799 \times 10^{9}$ & $-1.20232 \times 10^{8}$ & \pm 33436.3 & $\pm 10965.1 \mathrm{i}$ \\
\hline 39 & 0.0092158 & $1.21813 \times 10^{9}$ & $-1.29554 \times 10^{8}$ & \pm 34901.7 & $\pm 11382.2 \mathrm{i}$ \\
\hline 40 & 0.0092814 & $1.32531 \times 10^{9}$ & $-1.3931 \times 10^{8}$ & \pm 36404.8 & $\pm 11803.1 \mathrm{i}$ \\
\hline 41 & 0.0093437 & $1.43979 \times 10^{9}$ & $-1.49495 \times 10^{8}$ & \pm 37944.6 & $\pm 12226.8 \mathrm{i}$ \\
\hline 42 & 0.0094028 & $1.56277 \times 10^{9}$ & $-1.60161 \times 10^{8}$ & \pm 39531.8 & $\pm 12655.5 \mathrm{i}$ \\
\hline 43 & 0.0094591 & $1.69386 \times 10^{9}$ & $-1.71249 \times 10^{8}$ & \pm 41156.6 & $\pm 13086.2 \mathrm{i}$ \\
\hline 44 & 0.0095127 & $1.83418 \times 10^{9}$ & $-1.82797 \times 10^{8}$ & \pm 42827.3 & $\pm 13520.2 \mathrm{i}$ \\
\hline
\end{tabular}

Table 4: Stability of $\mathrm{L}_{2}$

\begin{tabular}{llllll}
\hline$\varphi^{\circ}$ & $\mathrm{L}_{2}(\mathrm{x})$ & $\Omega_{\mathrm{xx}}^{\circ}$ & $\Omega_{\mathrm{yy}}^{\mathrm{o}}$ & $\xi_{1,2}$ & \\
\hline 1 & 0.0121484 & 58.6428 & 2328.98 & \pm 48.1977 & $\xi_{3,4}$ \\
2 & 0.0121495 & 468.207 & 19393.1 & \pm 139.238 & \pm 2.66767 \\
$3-44$ & 0.0121496 & 1621.67 & 69142.3 & \pm 262.938 & \pm 40.2717 \\
\hline
\end{tabular}

Table 5: Stability of $\mathrm{L}_{3}$

\begin{tabular}{|c|c|c|c|c|c|}
\hline$\varphi^{\circ}$ & $\mathrm{L}_{3}(\mathrm{x})$ & $\Omega_{\mathrm{xx}}^{\mathrm{o}}$ & $\Omega_{\mathrm{yy}}^{\mathrm{o}}$ & $\xi_{1,2}$ & $\xi_{3,4}$ \\
\hline 1 & 0.1075610 & 73975.3 & -2321.2 & \pm 271.974 & $\pm 48.1807 \mathrm{i}$ \\
\hline 2 & 0.0603218 & 502793.1 & -18575.4 & \pm 709.075 & $\pm 136.292 \mathrm{i}$ \\
\hline 3 & 0.0447014 & $1.37909 \times 10^{6}$ & -62715 & \pm 1174.35 & $\pm 250.43 \mathrm{i}$ \\
\hline 5 & 0.0322547 & $4.36679 \times 10^{6}$ & -290447 & \pm 2089.69 & $\pm 538.932 \mathrm{i}$ \\
\hline 6 & 0.0291191 & $6.55878 \times 10^{6}$ & -501827 & \pm 2561.01 & $\pm 708.398 \mathrm{i}$ \\
\hline 7 & 0.0268565 & $9.35025 \times 10^{6}$ & -796700 & \pm 3057.82 & $\pm 892.581 \mathrm{i}$ \\
\hline 8 & 0.0251394 & $1.28577 \times 10^{7}$ & $-1.1887 \times 10^{6}$ & \pm 3585.76 & $\pm 1090.28 \mathrm{i}$ \\
\hline 9 & 0.0237881 & $1.72041 \times 10^{7}$ & $-1.69167 \times 10^{6}$ & \pm 4147.78 & $\pm 1300.64 \mathrm{i}$ \\
\hline 10 & 0.0226948 & $2.25035 \times 10^{7}$ & $-2.31889 \times 10^{6}$ & \pm 4743.78 & $\pm 1522.79 \mathrm{i}$ \\
\hline 11 & 0.0217913 & $2.88777 \times 10^{7}$ & $-3.08405 \times 10^{6}$ & \pm 5373.8 & $\pm 1756.15 \mathrm{i}$ \\
\hline 12 & 0.0210317 & $3.64435 \times 10^{7}$ & $-4.00042 \times 10^{6}$ & \pm 6036.84 & $\pm 2000.11 \mathrm{i}$ \\
\hline 13 & 0.0203840 & $4.53176 \times 10^{7}$ & $-5.08106 \times 10^{6}$ & \pm 6731.84 & $\pm 2254.12 \mathrm{i}$ \\
\hline 14 & 0.0198252 & $5.56215 \times 10^{7}$ & $-6.33912 \times 10^{6}$ & \pm 7457.98 & $\pm 2517.76 \mathrm{i}$ \\
\hline 15 & 0.0193383 & $6.74835 \times 10^{7}$ & $-7.78794 \times 10^{6}$ & \pm 8214.83 & $\pm 2790.69 \mathrm{i}$ \\
\hline 16 & 0.018911 & $8.11086 \times 10^{7}$ & $-9.44601 \times 10^{6}$ & \pm 9006.03 & $\pm 3073.44 \mathrm{i}$ \\
\hline 17 & 0.018531 & $9.63425 \times 10^{7}$ & $-1.13058 \times 10^{7}$ & \pm 9815.42 & $\pm 3362.42 \mathrm{i}$ \\
\hline 19 & 0.017889 & $1.32805 \times 10^{8}$ & $-1.57256 \times 10^{7}$ & \pm 11524.1 & $\pm 3965.55 \mathrm{i}$ \\
\hline 20 & 0.017616 & $1.54453 \times 10^{8}$ & $-1.83206 \times 10^{7}$ & \pm 12427.9 & $\pm 4280.26 \mathrm{i}$ \\
\hline 21 & 0.017368 & $1.78285 \times 10^{8}$ & $-2.11674 \times 10^{7}$ & \pm 13352.3 & $\pm 4600.81 \mathrm{i}$ \\
\hline 22 & 0.017143 & $2.04877 \times 10^{8}$ & $-2.43091 \times 10^{7}$ & \pm 14313.5 & $\pm 4930.43 \mathrm{i}$ \\
\hline 23 & 0.0169367 & $2.33850 \times 10^{8}$ & $-2.77178 \times 10^{7}$ & \pm 15292.2 & $\pm 5264.77 \mathrm{i}$ \\
\hline 24 & 0.0167477 & $2.65722 \times 10^{8}$ & $-3.14309 \times 10^{7}$ & \pm 16301.1 & $\pm 5606.32 \mathrm{i}$ \\
\hline 25 & 0.0165737 & $3.00518 \times 10^{8}$ & $-3.54488 \times 10^{7}$ & \pm 17335.4 & $\pm 5953.89 \mathrm{i}$ \\
\hline 26 & 0.0164132 & $3.38531 \times 10^{8}$ & $-3.97909 \times 10^{7}$ & \pm 18399.2 & $\pm 6308.01 \mathrm{i}$ \\
\hline 27 & 0.0162646 & $3.79853 \times 10^{8}$ & $-4.44607 \times 10^{7}$ & \pm 19489.8 & $\pm 6667.89 \mathrm{i}$ \\
\hline 28 & 0.0161267 & $4.24712 \times 10^{8}$ & $-4.94711 \times 10^{7}$ & \pm 20608.5 & $\pm 7033.57 \mathrm{i}$ \\
\hline 29 & 0.0159984 & $4.73294 \times 10^{8}$ & $-5.48305 \times 10^{7}$ & \pm 21755.3 & $\pm 7404.76 \mathrm{i}$ \\
\hline 30 & 0.0158787 & $5.25732 \times 10^{8}$ & $-6.05427 \times 10^{7}$ & \pm 22928.8 & $\pm 7780.92 \mathrm{i}$ \\
\hline 31 & 0.0157669 & $5.82401 \times 10^{8}$ & $-6.66277 \times 10^{7}$ & \pm 24133.1 & $\pm 8162.58 \mathrm{i}$ \\
\hline 32 & 0.0156622 & $6.43440 \times 10^{8}$ & $-7.30871 \times 10^{7}$ & \pm 25366.1 & $\pm 8549.10 \mathrm{i}$ \\
\hline 33 & 0.0155641 & $7.09356 \times 10^{8}$ & $-7.99477 \times 10^{7}$ & \pm 26633.7 & $\pm 8941.35 \mathrm{i}$ \\
\hline 34 & 0.0154717 & $7.79770 \times 10^{8}$ & $-8.71705 \times 10^{7}$ & \pm 27924.4 & $\pm 9336.51 \mathrm{i}$ \\
\hline 35 & 0.0153849 & $8.55785 \times 10^{8}$ & $-9.48224 \times 10^{7}$ & \pm 29253.8 & $\pm 9737.68 \mathrm{i}$ \\
\hline 38 & 0.0151526 & $1.11799 \times 10^{9}$ & $-1.20232 \times 10^{8}$ & \pm 33436.3 & $\pm 10965.1 \mathrm{i}$ \\
\hline 39 & 0.0150834 & $1.21813 \times 10^{9}$ & $-1.29554 \times 10^{8}$ & \pm 34901.7 & $\pm 11382.2 \mathrm{i}$ \\
\hline 40 & 0.0150178 & $1.32531 \times 10^{9}$ & $-1.39310 \times 10^{8}$ & \pm 36404.8 & $\pm 11803.1 \mathrm{i}$ \\
\hline 41 & 0.0149555 & $1.43979 \times 10^{9}$ & $-1.49495 \times 10^{8}$ & \pm 37944.6 & $\pm 12226.8 \mathrm{i}$ \\
\hline 42 & 0.0148964 & $1.56277 \times 10^{9}$ & $-1.60161 \times 10^{8}$ & \pm 39531.8 & $\pm 12655.5 \mathrm{i}$ \\
\hline 43 & 0.0148401 & $1.69386 \times 10^{9}$ & $-1.71249 \times 10^{8}$ & \pm 41156.6 & $\pm 13086.2 \mathrm{i}$ \\
\hline 44 & 0.0147865 & $1.83418 \times 10^{9}$ & $-1.82797 \times 10^{8}$ & \pm 42827.3 & $\pm 13520.2 \mathrm{i}$ \\
\hline
\end{tabular}

From the nature of the roots of characteristic Eqn. (11) as mentioned in Table 3, 4 and 5, it is observed that all the collinear libration points are unstable in $0^{\circ}<\varphi<45^{\circ}$.

\subsection{Stability of non-collinear libration points}

The roots of the characteristic Eqn. (11) i.e. $\xi_{i}(i=1,2,3,4)$ for $L_{4}$ are given in Table 6. 
Table 6: Stability of L

\begin{tabular}{|c|c|c|c|c|c|c|c|}
\hline$\varphi^{\circ}$ & $\mathrm{x}$ & $\mathrm{y}$ & $\Omega_{\mathrm{xx}}^{\mathrm{o}}$ & $\Omega_{\mathrm{yy}}^{\mathrm{o}}$ & $\Omega_{\mathrm{xy}}^{\mathrm{o}}$ & $\xi_{1,2}$ & $\xi_{3,4}$ \\
\hline 1 & 0.0121472 & -0.0181461 & 2320.87 & -4445427 & 1.88592 & \pm 48.1751 & $\pm 667.408 \mathrm{i}$ \\
\hline 2 & 0.0121493 & -0.00457585 & 18566.4 & -319586 & -2.37467 & \pm 136.257 & $\pm 565.324 \mathrm{i}$ \\
\hline 3 & 0.0121495 & -0.00201824 & 62657.1 & -343633 & -6.837 & \pm 250.312 & $\pm 586.206 \mathrm{i}$ \\
\hline 4 & 0.0121496 & -0.00112455 & 148495.01 & -513798 & $-4.09884 \times 10^{-5}$ & \pm 385.349 & $\pm 716.8 \mathrm{i}$ \\
\hline 5 & 0.0121496 & -0.000713569 & 289949 & -859493 & $-2.60087 \times 10^{-5}$ & \pm 538.468 & $\pm 927.091 \mathrm{i}$ \\
\hline 6 & 0.0121496 & -0.000492075 & 500843 & $-1.42622 \times 10^{6}$ & $-1.79355 \times 10^{-5}$ & \pm 707.702 & $\pm 1194.25 \mathrm{i}$ \\
\hline 7 & 0.0121496 & -0.000359556 & 794936 & $-2.26431 \times 10^{6}$ & $-1.31054 \times 10^{-5}$ & \pm 891.591 & $\pm 1504.76 \mathrm{i}$ \\
\hline 8 & 0.0121496 & -0.000274146 & $1.18591 \times 10^{6}$ & $-3.42512 \times 10^{6}$ & $-9.99229 \times 10^{-6}$ & \pm 1088.99 & $\pm 1850.71 \mathrm{i}$ \\
\hline 9 & 0.0121496 & -0.000215941 & $1.68737 \times 10^{6}$ & $-4.96004 \times 10^{6}$ & $-7.87079 \times 10^{-6}$ & \pm 1298.99 & $\pm 2227.12 \mathrm{i}$ \\
\hline 10 & 0.0121496 & -0.000174517 & $2.31286 \times 10^{6}$ & $-6.92016 \times 10^{6}$ & $-6.36093 \times 10^{-6}$ & \pm 1520.81 & $\pm 2630.62 \mathrm{i}$ \\
\hline 11 & 0.0121496 & -0.000143999 & $3.07571 \times 10^{6}$ & $-9.35701 \times 10^{6}$ & $-5.24859 \times 10^{-6}$ & \pm 1753.77 & $\pm 3058.92 \mathrm{i}$ \\
\hline 12 & 0.0121496 & -0.000120869 & $3.98932 \times 10^{6}$ & $-1.23219 \times 10^{7}$ & $-4.40553 \times 10^{-6}$ & \pm 1997.33 & $\pm 3510.25 \mathrm{i}$ \\
\hline 13 & 0.0121496 & -0.000102922 & $5.06679 \times 10^{6}$ & $-1.58672 \times 10^{7}$ & $-3.75138 \times 10^{-6}$ & \pm 2250.95 & $\pm 3983.36 \mathrm{i}$ \\
\hline 14 & 0.0121496 & -0.0000887176 & $6.32108 \times 10^{6}$ & $-2.00461 \times 10^{7}$ & $-3.23365 \times 10^{-6}$ & \pm 2514.17 & $\pm 4477.28 \mathrm{i}$ \\
\hline 15 & 0.0121496 & -0.0000772824 & $7.76512 \times 10^{6}$ & $-2.49123 \times 10^{7}$ & $-2.81685 \times 10^{-6}$ & \pm 2786.6 & $\pm 4991.23 \mathrm{i}$ \\
\hline 16 & 0.0121496 & -0.0000679405 & $9.41159 \times 10^{6}$ & $-3.05212 \times 10^{7}$ & $-2.47635 \times 10^{-6}$ & \pm 3067.83 & $\pm 5524.6 \mathrm{i}$ \\
\hline 17 & 0.0121496 & -0.0000602102 & $1.12731 \times 10^{7}$ & $-3.69289 \times 10^{7}$ & $-2.19459 \times 10^{-6}$ & \pm 3357.54 & $\pm 6076.92 \mathrm{i}$ \\
\hline 18 & 0.0121496 & -0.0000537409 & $1.33618 \times 10^{7}$ & $-4.41933 \times 10^{7}$ & $-1.95879 \times 10^{-6}$ & \pm 3655.38 & $\pm 6647.8 \mathrm{i}$ \\
\hline 19 & 0.0121496 & -0.0000482725 & $1.56901 \times 10^{7}$ & $-5.23738 \times 10^{7}$ & $-1.75947 \times 10^{-6}$ & \pm 3961.06 & $\pm 7236.97 \mathrm{i}$ \\
\hline 20 & 0.0121496 & -0.0000436085 & $1.82697 \times 10^{7}$ & $-6.15308 \times 10^{7}$ & $-1.58948 \times 10^{-6}$ & \pm 4274.31 & $\pm 7844.16 \mathrm{i}$ \\
\hline 21 & 0.0121496 & -0.0000395986 & $2.11125 \times 10^{7}$ & $-7.17275 \times 10^{7}$ & $-1.44332 \times 10^{-6}$ & \pm 4594.84 & $\pm 8469.21 \mathrm{i}$ \\
\hline 22 & 0.0121496 & -0.0000361259 & $2.42301 \times 10^{7}$ & $-8.30281 \times 10^{7}$ & $-1.31675 \times 10^{-6}$ & \pm 4922.41 & $\pm 9111.97 \mathrm{i}$ \\
\hline 23 & 0.0121496 & -0.0000330987 & $2.76332 \times 10^{7}$ & $-9.54999 \times 10^{7}$ & $-1.20641 \times 10^{-6}$ & \pm 5256.73 & $\pm 9772.41 \mathrm{i}$ \\
\hline 24 & 0.0121496 & -0.0000304438 & $3.13336 \times 10^{7}$ & $-1.0921 \times 10^{8}$ & $-1.10964 \times 10^{-6}$ & \pm 5597.64 & $\pm 10450.4 \mathrm{i}$ \\
\hline 25 & 0.0121496 & -0.0000281027 & $3.53415 \times 10^{7}$ & $-1.24232 \times 10^{8}$ & $-1.02431 \times 10^{-6}$ & \pm 5944.87 & $\pm 11145.9 \mathrm{i}$ \\
\hline 26 & 0.0121496 & -0.0000260278 & $3.96678 \times 10^{7}$ & $-1.40636 \times 10^{8}$ & $-9.48682 \times 10^{-7}$ & \pm 6298.24 & $\pm 11859.1 \mathrm{i}$ \\
\hline 27 & 0.0121496 & -0.0000241803 & $4.43226 \times 10^{7}$ & $-1.585 \times 10^{8}$ & $-8.81343 \times 10^{-7}$ & \pm 6657.52 & $\pm 12589.7 \mathrm{i}$ \\
\hline 28 & 0.0121496 & -0.0000225283 & $4.93151 \times 10^{7}$ & $-1.77906 \times 10^{8}$ & $-8.21129 \times 10^{-7}$ & \pm 7022.47 & $\pm 13338.1 \mathrm{i}$ \\
\hline 29 & 0.0121496 & -0.0000210451 & $5.46559 \times 10^{7}$ & $-1.98931 \times 10^{8}$ & $-7.67069 \times 10^{-7}$ & \pm 7392.97 & $\pm 14104.3 \mathrm{i}$ \\
\hline 30 & 0.0121496 & -0.0000197084 & $6.03558 \times 10^{7}$ & $-2.21657 \times 10^{8}$ & $-7.18348 \times 10^{-7}$ & \pm 7768.91 & $\pm 14888.1 \mathrm{i}$ \\
\hline 31 & 0.0121496 & -0.0000184998 & $6.64214 \times 10^{7}$ & $-2.46179 \times 10^{8}$ & $-6.74296 \times 10^{-7}$ & \pm 8149.93 & $\pm 15690.1 \mathrm{i}$ \\
\hline 32 & 0.0121496 & -0.0000174035 & $7.28614 \times 10^{7}$ & $-2.72591 \times 10^{8}$ & $-6.34337 \times 10^{-7}$ & \pm 8535.89 & $\pm 16510.3 \mathrm{i}$ \\
\hline 33 & 0.0121496 & -0.0000164059 & $7.96871 \times 10^{7}$ & $-3.00979 \times 10^{8}$ & $-5.97975 \times 10^{-7}$ & \pm 8926.76 & $\pm 17348.7 \mathrm{i}$ \\
\hline 34 & 0.0121496 & -0.0000154957 & $8.69041 \times 10^{7}$ & $-3.31451 \times 10^{8}$ & $-5.64801 \times 10^{-7}$ & \pm 9322.23 & $\pm 18205.8 \mathrm{i}$ \\
\hline 35 & 0.0121496 & -0.0000146631 & $9.45188 \times 10^{7}$ & $-3.64114 \times 10^{8}$ & $-5.34452 \times 10^{-7}$ & \pm 9722.08 & $\pm 19081.8 \mathrm{i}$ \\
\hline 36 & 0.0121496 & -0.0000138994 & $1.02544 \times 10^{8}$ & $-3.99065 \times 10^{8}$ & $-5.06616 \times 10^{-7}$ & \pm 10126.4 & $\pm 19976.6 \mathrm{i}$ \\
\hline 37 & 0.0121496 & -0.0000131974 & $1.10983 \times 10^{8}$ & $-4.36427 \times 10^{8}$ & $-4.81029 \times 10^{-7}$ & \pm 10534.8 & $\pm 20890.8 \mathrm{i}$ \\
\hline 38 & 0.0121496 & -0.0000125506 & $1.19846 \times 10^{8}$ & $-4.76311 \times 10^{8}$ & $-4.57454 \times 10^{-7}$ & \pm 10947.4 & $\pm 21824.5 \mathrm{i}$ \\
\hline 39 & 0.0121496 & -0.0000119535 & $1.29138 \times 10^{8}$ & $-5.18848 \times 10^{8}$ & $-4.35691 \times 10^{-7}$ & \pm 11363.9 & $\pm 22778.2 \mathrm{i}$ \\
\hline 40 & 0.0121496 & -0.0000114013 & $1.38859 \times 10^{8}$ & $-5.64185 \times 10^{8}$ & $-4.15564 \times 10^{-7}$ & \pm 11783.9 & $\pm 23752.6 \mathrm{i}$ \\
\hline 41 & 0.0121496 & -0.0000108895 & $1.49026 \times 10^{8}$ & $-6.12433 \times 10^{8}$ & $-3.96909 \times 10^{-7}$ & \pm 12207.6 & $\pm 24747.4 \mathrm{i}$ \\
\hline 42 & 0.0121496 & -0.0000104144 & $1.59639 \times 10^{8}$ & $-6.63751 \times 10^{8}$ & $-3.79592 \times 10^{-7}$ & \pm 12634.9 & $\pm 25763.4 \mathrm{i}$ \\
\hline 43 & 0.0121496 & -0.0000099726 & $1.70709 \times 10^{8}$ & $-7.18281 \times 10^{8}$ & $-3.63489 \times 10^{-7}$ & \pm 13065.5 & $\pm 26800.8 \mathrm{i}$ \\
\hline 44 & 0.0121496 & -0.0000095612 & $1.82233 \times 10^{8}$ & $-7.76204 \times 10^{8}$ & $-3.48494 \times 10^{-7}$ & \pm 13499.4 & $\pm 27860.4 \mathrm{i}$ \\
\hline
\end{tabular}

From the nature of the roots, it is observed that the non-collinear libration points are unstable. Similarly, $\mathrm{L}_{5}$ is also unstable.

\section{Conclusion}

In the present paper existence of collinear and non-collinear libration points for Earth-Moon system has been analyzed and this is found that all the libration points exist only in the interval $0^{\circ}<\varphi<$ $45^{\circ}$. There exist three collinear libration points and non-collinear libration points have been calculated by Newton-Raphson method taking initial values $x_{0}=\mu-1 / 2$ and $y_{0}= \pm \sqrt{3} / 2$ (Table 2). The noncollinear libration points are forming a right triangle with the primaries, right angled at the bigger primary. Further, all the libration points are unstable in $0^{\circ}<\varphi<45^{\circ}$.

\section{References}

[1] Abouelmagd, E. I., Asiri, H. M., Sharaf, M. A., 2013. "The effect of oblateness in the perturbed restricted three-body problem", Meccanica, vol. 48 (10), p. 2479-2490.http://dx.doi.org/10.1007/s11012-0139762-3.

[2] Byrd, P. F. \& Friedman, M. D., 1954. "Handbook of Elliptic Integrals for Engineers and Physicists", Springer-Verlag, Germany, p. 45.http://dx.doi.org/10.1007/978-3-642-52803-3.

[3] Idrisi, M. Javed, Taqvi, Z. A., 2013. "Restricted three-body problem when one of the primaries is an ellipsoid", Astrophysics and Space Science, vol. 348 (1), p. 41-56.http://dx.doi.org/10.1007/s10509-0131534-y.
[4] Idrisi, M. Javed, Taqvi, Z. A., 2014. "Existence and stability of the non-collinear libration points in the restricted three body problem when both the primaries are ellipsoid", Astrophysics and Space Science, vol. 350 (1), p. 133-141.http://dx.doi.org/10.1007/s10509-0131718-5.

[5] Idrisi, M. Javed, 2014. "Existence and stability of libration points in CR3BP when the smaller primary is an oblate spheroid", Astrophysics and Space Science, http://dx.doi.org/10.1007/s10509-014-2031-7.

[6] Khanna, M. \& Bhatnagar, K. B., 1999. "Existence and stability of libration points in the restricted three body problem when the smaller primary is a triaxial rigid body and the bigger one an oblate spheroid", Indian Journal of Pure and Applied Mathematics, vol. 30 (7), p. 721 733.

[7] Kumari, Reena, Kushvah, Badam Singh, 2014. "Stability regions of equilibrium points in restricted four-body problem with oblateness effects", Astrophysics and Space Science, vol. 349 (2), p. $693-$ 704.http://dx.doi.org/10.1007/s10509-013-1689-6.

[8] Narayan, A., Kumar, C. Ramesh, 2011, "Effects of photogravitational and oblateness on the triangular lagrangian points in the elliptical restricted three body problem", International Journal of Pure and Applied Mathematics, vol. 68(2), p. 201-224.

[9] Raheem, Abdul Razzaq Abdul, Singh, Jagdish, 2006, "Combined effects of perturbations, radiation and oblateness on the stability of equilibrium points in the restricted three-body problem", The Astronomical Journal, vol. 131, p. 1880 1885.http://dx.doi.org/10.1086/499300.

[10]Singh, Jagdish, Taura, J. J., 2013. "Motion in the generalized restricted three-body problem", Astrophysics and Space Science, vol. 343 (1) p. 95-106.http://dx.doi.org/10.1007/s10509-012-1225-0.

[11] Szebehely, Victor, 1967. "Theory of Orbits, The restricted problem of three bodies", Academic Press, New York and London. 


\section{Appendix I}

$$
\begin{aligned}
& \Omega_{x x}=n^{2}-\frac{\mu}{r_{2}^{3}}+\frac{3 \mu}{r_{2}^{5}}(x+1-\mu)^{2} \\
& -3(1-\mu)\left[\frac{E(\varphi, k)-F(\varphi, k)}{p_{6} p_{8}}\right. \\
& -\left\{1-k^{2} \sin ^{2} \varphi \frac{(x-\mu)^{2}}{p_{6}}\right. \\
& \left.+\left(\frac{1}{p_{6}}+\frac{1-k^{2} \sin ^{2} \varphi}{p_{9}}\right) y^{2}\right\} \\
& \times \frac{\left(\lambda+p_{3}\right)}{2\left(\lambda+a^{2}\right)\left(2 \lambda+p_{1}-r_{1}^{2}\right) \sqrt{\lambda+c^{2}} \sqrt{1-k^{2} \sin ^{2} \varphi}} \\
& \left.-\frac{\left(\lambda^{2}+2 c^{2} \lambda+p_{11}\right)\left(\lambda+p_{3}\right) y^{2}}{2 p_{7}\left(2 \lambda+p_{1}-r_{1}^{2}\right) \sqrt{\lambda+c^{2}}\left(\lambda^{2}+p_{5} \lambda+p_{10}\right)^{\frac{3}{2}}}\right] \\
& -3(1-\mu)(x-\mu) \\
& {\left[A_{1}-\frac{\left(\lambda+p_{3}\right) A_{2}}{2\left(\lambda+a^{2}\right)\left(2 \lambda+p_{1}-r_{1}^{2}\right) \sqrt{\lambda+c^{2}} \sqrt{1-k^{2} \sin ^{2} \varphi}}\right.} \\
& \left\{1-k^{2} \sin ^{2} \varphi \frac{(x-\mu)^{2}}{p_{6}}\right. \\
& \left.\left.+\left(\frac{1}{p_{6}}+\frac{1-k^{2} \sin ^{2} \varphi}{p_{9}}\right) y^{2}\right\} A_{3}-A_{4}\right]
\end{aligned}
$$$$
\Omega_{x y}=\frac{3 \mu}{r_{2}^{5}}(x+1-\mu) \quad y-3(1-\mu)(x-\mu)
$$$$
\left[A_{5}-\frac{\left(\lambda+p_{3}\right) A_{6}}{2\left(\lambda+a^{2}\right)\left(2 \lambda+p_{1}-r_{1}^{2}\right) \sqrt{\lambda+c^{2}} \sqrt{1-k^{2} \sin ^{2} \varphi}}\right.
$$$$
-\left\{1-k^{2} \sin ^{2} \varphi \frac{(x-\mu)^{2}}{p_{6}}+\left(\frac{1}{p_{6}}+\frac{1-k^{2} \sin ^{2} \varphi}{p_{9}}\right) y^{2}\right\} A_{7}
$$$$
-A_{8} y^{2}-
$$$$
\left.\frac{\left(\lambda^{2}+2 c^{2} \lambda+p_{11}\right)\left(\lambda+p_{3}\right) y}{p_{7}\left(2 \lambda+p_{1}-r_{1}^{2}\right) \sqrt{\lambda+c^{2}}\left(\lambda^{2}+p_{5} \lambda+p_{10}\right)^{\frac{3}{2}}}\right],
$$

$$
\begin{aligned}
& \Omega_{y y}=n^{2}-\frac{\mu}{r_{2}^{3}}+\frac{3 \mu}{r_{2}^{5}} y^{2}-3(1-\mu)\left[\frac{1}{p_{8}}\left(\frac{E(\varphi, k)}{p_{9}}+\frac{F(\varphi, k)}{p_{6}}\right)\right. \\
& -\left\{1-k^{2} \sin ^{2} \varphi \frac{(x-\mu)^{2}}{p_{6}}+\right. \\
& \left.\left(\frac{1}{p_{6}}+\frac{1-k^{2} \sin ^{2} \varphi}{p_{9}}\right) y^{2}\right\} \\
& \times \frac{\left(\lambda+p_{4}\right)}{2\left(\lambda+a^{2}\right)\left(2 \lambda+p_{1}-r_{1}^{2}\right) \sqrt{\lambda+c^{2}} \sqrt{1-k^{2} \sin ^{2} \varphi}}- \\
& \left.\frac{\left(\lambda^{2}+2 c^{2} \lambda+p_{11}\right)\left(\lambda+p_{4}\right) y^{2}}{2 p_{7}\left(2 \lambda+p_{1}-r_{1}^{2}\right) \sqrt{\lambda+c^{2}}\left(\lambda^{2}+p_{5} \lambda+p_{10}\right)^{\frac{3}{2}}}\right]- \\
& 3(1-\mu) y\left[\begin{array}{l}
A_{9} \\
-A_{6} \frac{\left(\lambda+p_{4}\right)}{2\left(\lambda+a^{2}\right)\left(2 \lambda+p_{1}-r_{1}^{2}\right) \sqrt{\lambda+c^{2}} \sqrt{1-k^{2} \sin ^{2} \varphi}}
\end{array}\right. \\
& \left\{1-k^{2} \sin ^{2} \varphi \frac{(x-\mu)^{2}}{p_{6}}+\left(\frac{1}{p_{6}}+\frac{1-k^{2} \sin ^{2} \varphi}{p_{9}}\right) y^{2}\right\} A_{10}- \\
& \left.\frac{\left(\lambda^{2}+2 c^{2} \lambda+p_{11}\right)\left(\lambda+p_{4}\right) y}{p_{7}\left(2 \lambda+p_{1}-r_{1}^{2}\right) \sqrt{\lambda+c^{2}}\left(\lambda^{2}+p_{5} \lambda+p_{10}\right)^{\frac{3}{2}}}-A_{11} y^{2}+A_{12}\right] \text {, }
\end{aligned}
$$

where

$$
\begin{aligned}
& A_{1}=\frac{(x-\mu)\left(\lambda+p_{3}\right) k^{2} \sin ^{2} \varphi}{p_{6}\left(\lambda+a^{2}\right)\left(2 \lambda+p_{1}-r_{1}^{2}\right) \sqrt{\lambda+c^{2}} \sqrt{1-k^{2} \sin ^{2} \varphi}}, \\
& A_{2}=\frac{p_{8}(x-\mu)\left(\lambda+p_{3}\right) k^{2} \sin 2 \varphi}{\left(\lambda+a^{2}\right)\left(2 \lambda+p_{1}-r_{1}^{2}\right) \sqrt{\lambda+c^{2}}}\left(\frac{(x-\mu)^{2}}{p_{6}}+\frac{y^{2}}{p_{9}}\right)-\frac{2 k^{2}(x-\mu) \sin ^{2} \varphi}{p_{6}},
\end{aligned}
$$$$
A_{3}=\frac{(x-\mu)\left(\lambda+p_{3}\right)}{\left(\lambda+a^{2}\right)\left(2 \lambda+p_{1}-r_{1}^{2}\right)^{2} \sqrt{\lambda+c^{2}} \sqrt{1-k^{2} \sin ^{2} \varphi}}
$$$$
\left[1-\frac{\lambda+p_{3}}{\lambda+a^{2}}-\left(\frac{2\left(\lambda+p_{3}\right)}{2 \lambda+p_{1}-r_{1}^{2}}-1\right)-\frac{\lambda+p_{3}}{2\left(\lambda+c^{2}\right)}\right.
$$$$
\left.-\frac{p_{8}\left(\lambda+p_{3}\right) k^{2} \sin 2 \varphi}{4\left(\lambda+a^{2}\right)\left(1-k^{2} \sin ^{2} \varphi\right) \sqrt{\lambda+c^{2}}}\right] \text {, }
$$$$
\begin{aligned}
& A_{4}=\frac{y^{2}(x-\mu)\left(\lambda+p_{3}\right)}{2 p_{7} \sqrt{\lambda+c^{2}}\left(2 \lambda+p_{1}-r_{1}^{2}\right)^{2}\left(\lambda^{2}+p_{5} \lambda+p_{10}\right)^{\frac{3}{2}}} \\
& {\left[4\left(\lambda+c^{2}\right)\left(\lambda+p_{3}\right)+2\left(\lambda^{2}+2 c^{2} \lambda+p_{11}\right)-\right.}
\end{aligned}
$$

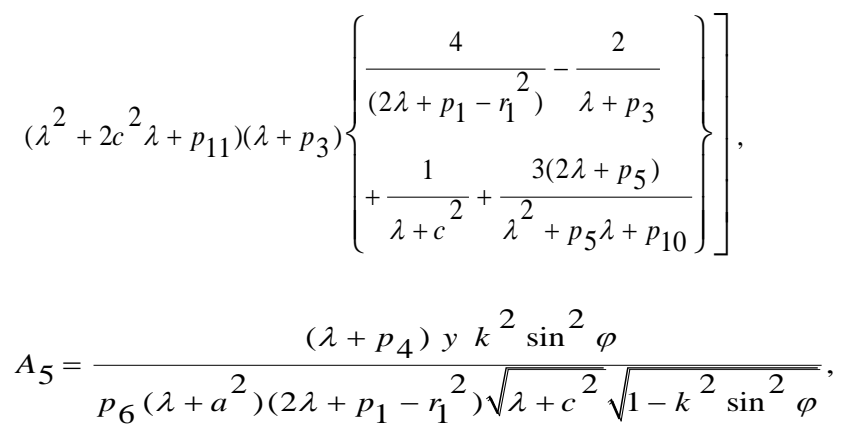




$$
\begin{aligned}
& A_{6}=\frac{p_{8}\left(\lambda+p_{4}\right) y k^{2} \sin 2 \varphi}{\left(\lambda+a^{2}\right)\left(2 \lambda+p_{1}-r_{1}^{2}\right) \sqrt{\lambda+c^{2}}}\left(\frac{(x-\mu)^{2}}{p_{6}}+\frac{y^{2}}{p_{9}}\right) \\
& +2 y\left(\frac{1}{p_{6}}+\frac{1-k^{2} \sin ^{2} \varphi}{p_{9}}\right),
\end{aligned}
$$

$$
\begin{aligned}
& A_{7}=\frac{\left(\lambda+p_{4}\right) y}{\left(\lambda+a^{2}\right)\left(2 \lambda+p_{1}-r_{1}^{2}\right)^{2} \sqrt{\lambda+c^{2}} \sqrt{1-k^{2} \sin ^{2} \varphi}} \\
& {\left[1-\frac{\lambda+p_{3}}{\lambda+a^{2}}-\left(\frac{2}{2 \lambda+p_{1}-r_{1}^{2}}-\frac{1}{\lambda+p_{4}}\right)\left(\lambda+p_{3}\right)\right.}
\end{aligned}
$$$$
\left.-\frac{\lambda+p_{3}}{2\left(\lambda+c^{2}\right)}-\frac{p_{8}\left(\lambda+p_{3}\right) k^{2} \sin 2 \varphi}{4\left(\lambda+a^{2}\right) \sqrt{\lambda+c^{2}}\left(1-k^{2} \sin ^{2} \varphi\right)}\right],
$$$$
A_{8}=\frac{y}{2 p_{7} \sqrt{\lambda+c^{2}}\left(2 \lambda+p_{1}-r_{1}^{2}\right)^{2}\left(\lambda^{2}+p_{5} \lambda+p_{10}\right)^{\frac{3}{2}}}
$$$$
\left[2\left(2 \lambda+p_{1}-r_{1}^{2}\right)\left(\lambda+p_{3}\right)\left(\lambda^{2}+2 c^{2} \lambda+p_{11}\right)+\right.
$$$$
2\left(\lambda+p_{4}\right) y^{2}\left\{2\left(\lambda+c^{2}\right)\left(\lambda+p_{3}\right)+\left(\lambda^{2}+2 c^{2} \lambda+p_{11}\right)\right\}
$$$$
-\left(\lambda^{2}+2 c^{2} \lambda+p_{11}\right)\left(\lambda+p_{3}\right)\left(\lambda+p_{4}\right) y^{2} \times
$$$$
\left.\left\{\frac{4}{\left(2 \lambda+p_{1}-r_{1}^{2}\right)}-\frac{2}{\lambda+p_{4}}+\frac{1}{\lambda+c^{2}}+\frac{3\left(2 \lambda+p_{5}\right)}{\lambda^{2}+p_{5} \lambda+p_{10}}\right\}\right] \text {, }
$$

$A_{9}=\frac{-y\left(\lambda+p_{4}\right)}{\left(\lambda+a^{2}\right)\left(2 \lambda+p_{1}-r_{1}^{2}\right) \sqrt{\lambda+c^{2}} \sqrt{1-k^{2} \sin ^{2} \varphi}}$ $\left[\frac{1}{p_{6}}+\frac{1-k^{2} \sin ^{2} \varphi}{p_{9}}\right]$,

$$
\begin{aligned}
& A_{10}=\frac{\left(\lambda+p_{4}\right) y}{\left(\lambda+a^{2}\right)\left(2 \lambda+p_{1}-r_{1}^{2}\right)^{2} \sqrt{\lambda+c^{2}} \sqrt{1-k^{2} \sin ^{2} \varphi}} \\
& {\left[1-\frac{\lambda+p_{4}}{\lambda+a^{2}}-\left(\frac{\lambda+p_{4}}{2 \lambda+p_{1}-r_{1}^{2}}-1\right)\right.} \\
& \left.-\frac{\lambda+p_{4}}{2\left(\lambda+c^{2}\right)}-\frac{p_{8}\left(\lambda+p_{4}\right) k^{2} \sin 2 \varphi}{4\left(\lambda+a^{2}\right)\left(1-k^{2} \sin ^{2} \varphi\right) \sqrt{\lambda+c^{2}}}\right],
\end{aligned}
$$

$$
A_{11}=\frac{y\left(\lambda+p_{4}\right)}{p_{7}\left(2 \lambda+p_{1}-r_{1}^{2}\right)^{2} \sqrt{\lambda+c^{2}}\left(\lambda^{2}+p_{5} \lambda+p_{10}\right)^{\frac{3}{2}}} \times
$$

$$
\begin{aligned}
& {\left[\begin{array}{l}
\left(2 \lambda+p_{1}-(x-\mu)^{2}\right)\left(\lambda^{2}+2 c^{2} \lambda+p_{11}\right) \\
+2 y^{2}\left(\lambda+c^{2}\right)\left(\lambda+p_{4}\right)-\left(\lambda^{2}+2 c^{2} \lambda+p_{11}\right)\left(\lambda+p_{4}\right) y^{2} \\
\left\{\frac{2}{\left(2 \lambda+p_{1}-r_{1}^{2}\right)}-\frac{1}{\left(\lambda+p_{4}\right)}+\frac{1}{2\left(\lambda+c^{2}\right)}+\frac{3\left(2 \lambda+p_{5}\right)}{2\left(\lambda^{2}+p_{5} \lambda+p_{10}\right)}\right\}
\end{array}\right],} \\
& A_{12}=\frac{y\left(\lambda+p_{4}\right)\left[\lambda^{2}+p_{5} \lambda+p_{10}-\left(\lambda+c^{2}\right)\left(2 \lambda+p_{5}\right)\right]}{p_{7}\left(2 \lambda+p_{1}-r_{1}^{2}\right) \sqrt{\lambda+c^{2}}\left(\lambda^{2}+p_{5} \lambda+p_{10}\right)^{\frac{3}{2}}} \text {. }
\end{aligned}
$$

